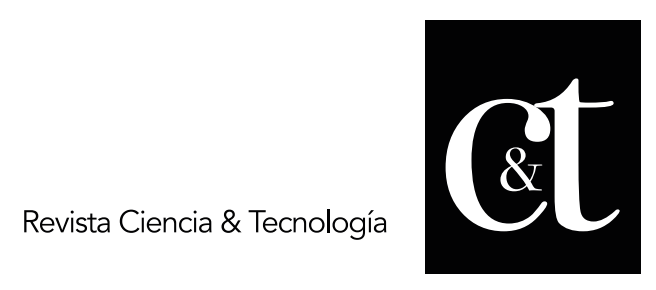

No. 32, 31 de octubre de 2021

ISSN impreso: 1390 - 6321

ISSN online: 2661 - 6734

\title{
Inbound marketing y reactivación económica de las pymes gimnasios de Babahoyo
}

\section{Inbound marketing and economic reactivation of SMEs Babahoyo gyms}

\author{
Andrés Hernández Lavayen ${ }^{1}$ \\ ahernandez@uteg.edu.ec \\ https://orcid.org/0000-0003-4741-1547 \\ Jaime Villalva Ronquillo 2 \\ jaivill r@hotmail.com \\ https://orcid.org/0000-0003-2423-3506
}

Recibido: 28/6/2021, Aceptado: 28/9/2021

\begin{abstract}
RESUMEN
El presente estudio aborda la problemática que afectó al sector gimnasios debido a la pandemia por Covid-19. Esta industria atravesó por una situación muy compleja, que desencadenó el cierre definitivo de algunos establecimientos; y en otros casos el proceso de reactivación económica ha sido muy lento, por lo que se estudió la estrategia de inbound marketing, con el objetivo de conocer su aporte en la gestión de actividades de gimnasios. El estudio es de tipo no experimental, transversal, se realiza a través de un estudio exploratorio, descriptivo y utilizando los métodos deductivo e inductivo. Se tomó como muestra a gimnasios de pequeña, mediana y gran capacidad de la zona urbana de la ciudad de Babahoyo, con recolección de datos mediante encuesta digital. Se concluye que las estrategias de inbound marketing aportan a la reactivación económica de los gimnasios de la ciudad de Babahoyo; identificando a la creación de contenidos como una herramienta que los gimnasios utilizan para generar una imagen de autoridad en su nicho de mercado y que los contenidos buscan que los clientes se identifiquen con la marca y su fidelización.
\end{abstract}

Palabras clave: Gimnasios, Inbound Marketing, Marketing de Contenidos, Estrategia, Reactivación

\section{ABSTRACT}

This study addresses the problem that affected the gym sector due to the Covid19 pandemic. This industry went through a very complex situation, which triggered the definitive closure of some establishments; and in other cases, the economic reactivation process has been very slow, so the inbound marketing strategy was studied, with the aim of knowing its contribution in the management of gym activities. The study is of a non-experimental, cross-sectional type, it is carried out through an exploratory, descriptive study and using deductive and inductive methods. Small, medium and large capacity gyms in the urban area of the city of Babahoyo were taken as a sample, with data collection through a digital survey.

${ }^{1}$ Magíster en Marketing y Ventas, Universidad Tecnológica Empresarial de Guayaquil, Ecuador

${ }^{2}$ Magíster en Administración de Empresas, Universidad Tecnológica Empresarial de Guayaquil, Ecuador 


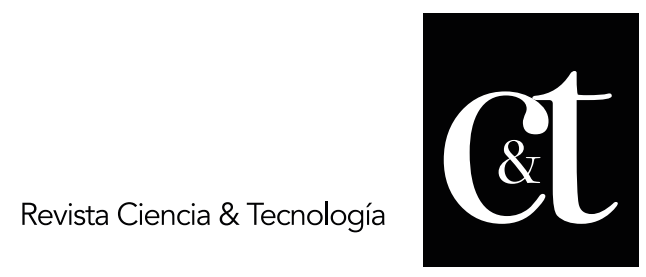

No. 32, 31 de octubre de 2021

ISSN impreso: 1390 - 6321

ISSN online: 2661 - 6734

It is concluded that inbound marketing strategies contribute to the economic reactivation of gyms in the city of Babahoyo; identifying the creation of content as a tool that gyms use to generate an image of authority in their market niche and that the content seeks that customers identify with the brand and its loyalty.

Keywords: Gyms, Inbound Marketing, Content Marketing, Strategy, Reactivation

\section{Introducción}

En el Ecuador la población de doce años y más, que realiza alguna actividad física, se establece en $\$ 5^{\prime} 043.516$; de los cuales aproximadamente 257.219 personas asisten con frecuencia a un gimnasio, de acuerdo a la encuesta condiciones de Vida ECV - Sexta Ronda 2015 del Instituto Nacional de Estadística y Censos (2015). En relación al impacto de la industria en la que se agrupan las actividades de gimnasios sobre el producto interno bruto, éste representó al año 2019, $\$ 9.738,50$ millones de dólares, de acuerdo al Banco Central del Ecuador (2020). De acuerdo con lo expuesto por la Organización Mundial de la Salud, la falta de actividad física es un factor de riesgo considerable para las enfermedades no transmisibles, como los accidentes cerebrovasculares, la diabetes y el cáncer (OMS, 2017). Este organismo mundial, hace un llamado a buscar estrategias para reducir la inactividad hasta el 2025 , al menos en un $10 \%$ en la población.

\section{El Inbound Marketing como estrategia de negocio en la industria de los gimnasios}

Para Loredana (2016) "el inbound marketing es una forma de marketing que requiere una orientación muy cuidadosa de la audiencia y una comunicación personalizada con ella a través de contenido de alta calidad" (p. 62). Mientras que para Raatikainen (2018), el inbound marketing se enfoca en llevar a la audiencia a su material en lugar de que sea al revés, por lo que se requiere, que el especialista en marketing piense hacia dónde se dirige su audiencia y cómo buscan cosas. Desde el punto de vista de Thi Thuy (2020) "el inbound marketing es una estrategia que utiliza contenidos apropiados y llamativos que están hechos a medida para adaptarse a las necesidades o requisitos de los clientes" (p. 24).

El inbound marketing nace como una respuesta a los problemas del marketing tradicional o outbound marketing cuyo fin es exponerse al usuario para poder ser encontrado por el mismo de forma no invasiva. Se basa en la atracción por medios que le aporten conocimiento, curiosidades y la posibilidad de participación en los mismos y así captar su atención. Básicamente lo que se hace es crear contenido de calidad en función de lo demandado por un determinado perfil, es decir, por una audiencia o comunidad que buscan esa información en particular (Teba Garrido, 2016). En otras palabras, el inbound marketing es el que consiste en ofrecer al cliente lo que le interesa a través de una comunicación bidireccional que causa beneficios mutuos: para el consumidor y para la empresa. Así el marketing digital se destaca primordialmente por crear una relación de lealtad y confianza con los usuarios, por lo que, el inbound marketing "se convierte en el aspecto principal del marketing digital" (Loredana, 2016, p. 62). 


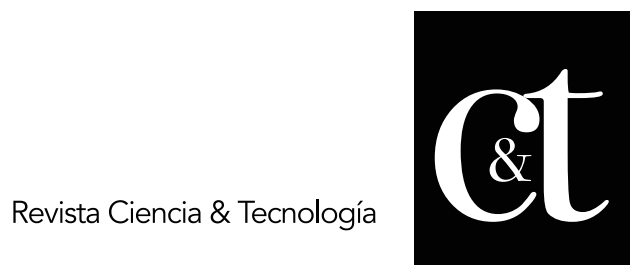

No. 32, 31 de octubre de 2021

ISSN impreso: 1390 - 6321

ISSN online: 2661 - 6734

De esa manera se permite mantener una comunicación inteligente y abierta con los usuarios que dirigieron su atención a los servicios y productos de la empresa hasta lograr obtener una relación personal con ellos, por medio de la promoción de contenidos enfocados a sus requerimientos.

\section{Modelo de metodología de Inbound Marketing}

Uno de los modelos del inbound marketing, es el creado por HubSpot, que explica la metodología y sus herramientas, cuyos elementos implícitos son: atraer, involucrar y deleitar para crear valor y confianza a los clientes de la empresa. El propósito del modelo es agregar valor en cada paso del viaje del cliente con el propósito de crear relaciones duraderas y satisfactorias. Al usar este modelo, se busca la expansión del negocio, además de la retención de más clientes satisfechos, y además crear un entorno de referenciación de boca en boca. Este método hará que el negocio aumente la satisfacción de los clientes, ya sea mediante la recompra o la promoción de la empresa que creará nuevos clientes. Cada paso de la metodología de inbound marketing se debe analizar para saber su efectividad y lo que se necesita cambiar. (HubSpot, 2020)

Atraer es el primer desafío al que se enfrentan las empresas y los comercializadores en el proceso de inbound marketing. Según Hubspot (2020), la metodología del inbound marketing no es atraer a la mayor cantidad de la audiencia posible. En otras palabras, las empresas no deberían intentar atraer a todos, pero si deben atraer a las personas adecuadas, en el momento adecuado y en el lugar adecuado. Otra forma de decirlo, es encontrar respuestas a las preguntas: quién, cuándo y dónde (Cintell, 2019).

Las empresas necesariamente deben atraer personas que puedan convertirse en clientes potenciales y luego en clientes satisfechos, esto se logra creando contenido valioso, en el momento adecuado y cuando los clientes lo requieren. Por lo que la empresa debe mantenerse activa en las redes sociales, mediante la creación de publicaciones de blog y contenido multimedia; además, debe aumentar el conocimiento de la marca mediante la creación de anuncios que se dirijan a clientes potenciales (Lindblom \& Andreasson, 2019).

Mediante el proceso de atracción los visitantes conocen la empresa y sus productos o servicios ofrecidos, se ha recopilado información preliminar sobre el producto e identificaron varios detalles sobre lo que las empresas tienen para ofrecer. Ahora, las empresas necesitan convertirlos, de un extraño, en clientes potenciales que son prospectos reales, dispuestos a comprar bienes o servicios. Establecer relaciones con extraños es una de las tareas más delicadas, y no es un trabajo de un día, generalmente requiere un tiempo y esfuerzo considerable por parte de las empresas (Thi Thuy, 2020). La relación debe construirse de manera constante, y debe ser un contacto directo para obtener mejores resultados. Como parte del intercambio de datos de contacto o información personal, las empresas deben ofrecer algo que los clientes consideren como valor percibido.

El deleite, del cliente al promotor, este es el objetivo primordial, cuando los especialistas en marketing pasan al cuarto paso, el último de todo el inbound proceso de marketing. La satisfacción del cliente es un procedimiento para llevar experiencia del cliente a un nivel superior. 


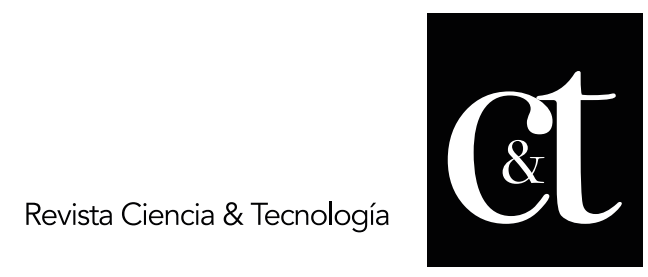

No. 32, 31 de octubre de 2021

ISSN impreso: 1390 - 6321

ISSN online: 2661 - 6734

No se trata solo de satisfacer su demanda sino de superar sus expectativas para que las empresas puedan crear una experiencia de cliente positiva y mantener relaciones a largo plazo con ellos. En este contexto, para distribuir contenido relevante a los clientes, es preciso realizarlo en el momento perfecto, para ello, se pueden utilizar herramientas de automatización de tiempo, correo electrónico y marketing, combinando herramientas de conversación con el equipo de ventas. Es posible utilizar una variación de diferentes formas de contenido que el grupo objetivo prefiere, como los videos, publicación de contenido valioso que puedan ser compartidos (HubSpot, 2020).

\section{Target y Buyer persona}

En los últimos tiempos, las estrategias de marketing se han dirigido a ciertos grupos de compradores de interés para la marca conocidos como targets, alejándose del marketing para todos y segmentando por grupos de usuarios (Nuviala Nuviala et al., 2014) pero en los últimos años este paradigma está cambiando. Por tanto, dada esa intensa competencia, ahora no sólo es necesario tener claro cuál es el target, sino que también se debe identificar dentro de él al comprador: buyer persona y combinar ambos aspectos, de forma que se intente disminuir todo lo posible la incertidumbre que pueda tener el consumidor en favor de la marca. Este buyer persona es: la descripción sociodemográfica de un perfil de comprador específico, con determinadas características e intereses, por lo que este nuevo enfoque permite ofrecer una información más individualizada, para crear un contenido más adaptado y por tanto mejor para el consumidor en cada una de sus fases de compra (Alguacil et al, 2020).

\section{Marketing de contenidos}

Debido a la vertiginosa revolución de Internet, el marketing tuvo que cambiar y adaptarse a él. Los consumidores son más cautelosos y escépticos hacia los mensajes de marketing que reciben hoy y ya no están tan persuadidos por los anuncios tradicionales como antes (Repovienè, 2017). El marketing de contenidos es el elemento principal del inbound marketing. El contenido debe ser confiable, actualizado, interesante, valioso, útil, etc. El marketing de contenidos, es hoy en día, uno de los métodos más destacados en lo que respecta a los procesos de marketing y su valor ha aumentado con el tiempo, debido a la digitalización que ha creado nuevas comunicaciones.

Distribuye contenido valioso para ayudar a los clientes a comprender los problemas, facilite conceptos complicados o proporcione valor a través de información útil. (Repoviené, 2017). Un contenido de calidad, debe cumplir con varios requisitos: debe dirigirse a una audiencia específica, posee un objetivo concreto, tiene credibilidad, es útil, y consigue ser compartido. Sobre la difusión del contenido, Maciá (2013) afirma que las empresas pueden favorecer la viralidad de sus contenidos si son capaces de seguir un sencillo proceso.

\section{Métricas de inbound marketing}

El inbound marketing se basa en los datos y en cómo se utilizan. Aprovechando la data para su ventaja es crucial, porque se necesita saber qué tipo de contenido funciona, quién ve ese contenido con información valiosa y cómo puede apoyar eficazmente a los clientes potenciales hacia un resultado mutuamente posible. (Hubspot 2020). 


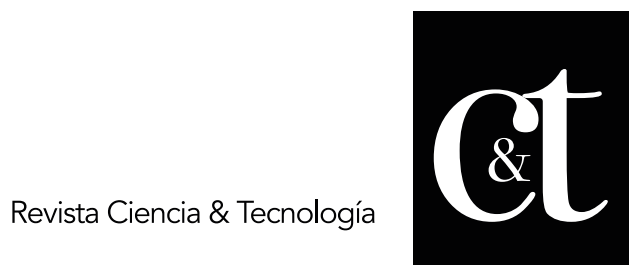

No. 32, 31 de octubre de 2021

ISSN impreso: 1390 - 6321

ISSN online: 2661 - 6734

La eficacia del marketing también se puede medir en recursos gastados como dinero y tiempo, y cuánto generó en nuevos ingresos, en comparación con los recursos usados, lo que se conoce como retorno sobre la inversión. Diferentes métodos de marketing tienen una variación en su ROI ya que algunos tienen más potencial para generar ingresos con menores recursos.

EI CAC se puede calcular de manera similar al costo por cliente potencial, pero cambia el número de clientes potenciales al número de clientes adquiridos. Este número refleja también cuán efectivas son las ventas, pero también considerando los esfuerzos de marketing, convirtiendo clientes potenciales en los clientes pago. Sobre todo, refleja el proceso de adquisición de clientes, en vez del costo por cliente potencial (Chase, 2020).

En la toma de decisiones de alto nivel, es importante saber la efectividad de la asignación de recursos en marketing. Para tomar decisiones correctas, las organizaciones deben ser capaces de comparar diferentes procesos juntos y el ROI es uno de los más universales con el que se puede calcular para casi todas las actividades comerciales. EI ROI de marketing se puede calcular con un par de métodos diferentes. Uno es para calcular cuántos clientes nuevos se adquirieron durante cierto período, multiplicado con el valor promedio para el cliente. El valor del cliente puede ser calculado contando la suma promedio que un cliente paga durante la compra.

\section{Metodología}

La presente investigación cuali-cuantitativa es de tipo no experimental transversal y de acuerdo a la clasificación expuesta por Hernández et al. (2014), se realizó a través de un estudio exploratorio y descriptivo. Se utilizó una metodología cuantitativa para estudiar las estrategias de inbound marketing. Se utilizó el método inductivo con el fin de explorar, describir, y luego generar perspectivas teóricas sobre la identificación de herramientas tecnológicas más efectivas y posteriormente reconocer el nivel de factibilidad en la implementación de estrategias inbound marketing en los negocios de gestión de actividades de gimnasio. También se emplea el método deductivo a través del cual se desciende de las teorías más generales hasta las más particulares, partiendo de enunciados de carácter general se infieren enunciados particulares. Se consideró como objeto de análisis los negocios de gestión de actividades de gimnasio de la ciudad de Babahoyo, donde existen 22 establecimientos asentados en toda la zona urbana, cuya población mayor a 12 años que ejerce algún tipo de actividad física o deportiva, asciende a 12.160 personas, de conformidad a la encuesta de uso de tiempo de los individuos y de tiempo libre, formulada por el INEC (2012). Fueron seleccionados tres gimnasios de la ciudad de Babahoyo, de acuerdo con los siguientes criterios de inclusión: diferentes tamaños de negocio, uno pequeño, otro mediano y uno grande; y, que deben encontrarse ubicados en la zona urbana, al norte, centro y sur de la ciudad. Para efectos de contrastar las variaciones en función de la línea de tiempo, se efectúa el análisis antes, durante y después de la suspensión de actividades dispuestas por las autoridades de control.

En relación a las percepciones de las estrategias inbound marketing, son medidas a través de un cuestionario multifactor, que es aplicable a través de una encuesta diseñada para medir las variables según las dimensiones consideradas. 


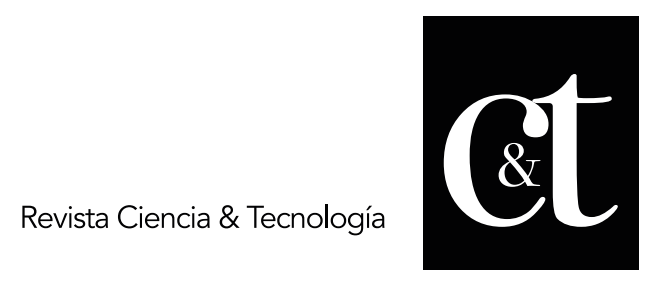

No. 32, 31 de octubre de 2021

ISSN impreso: 1390 - 6321

ISSN online: 2661 - 6734

Con el propósito de examinar la validez de constructo de las escalas empleadas en el presente estudio se realizará un análisis descriptivo e inferencial. A través de la revisión en fuentes primarias, se recabará información referente a las variables en estudio. Para recolectar los datos, la encuesta se digitalizó a través de una herramienta en línea denominada Zoho Forms. Los datos recabados son sometidos a un proceso de análisis estadístico utilizando Microsoft Excel.

\section{Resultados y discusión}

Los gimnasios fueron gravemente afectados por la declaratoria de suspensión de actividades debido a la pandemia por covid-19. Aproximadamente por 6 meses los gimnasios facturaban en contra, con egresos que superaban los pocos ingresos que generaban en estos meses, donde no se brindó el servicio con atención presencial. Sin embargo, los gastos que se generaron durante este mismo tiempo, se derivaron de los compromisos adquiridos previamente y adecuaciones para el cumplimiento de las normas de bioseguridad exigidas por los organismos de control, particularmente por el Gobierno Autónomo Descentralizado de la ciudad. Por otra parte, posterior a la suspensión de actividades de gimnasios, y una vez que se reactiva de forma parcial el funcionamiento de estos negocios, se reflejan menos utilidades con respecto a las percibidas antes de la suspensión de actividades, reduciéndose en aproximadamente un $40 \%$, esto se debe a que se vienen generando varias políticas y disposiciones que limitan el funcionamiento de los gimnasios, reducción de los horarios de atención, reducción del aforo, restringir el uso de máquinas, equipos, saunas, entre otros que representen un foco de contagio. Adicionalmente a estas restricciones, existe una campaña informativa que ubica a los gimnasios como un establecimiento de posible contagio, lo que ha causado temor en los usuarios, que, por evitar contagiarse, dejan de asistir a las instalaciones.

Tabla 1: Utilidades generadas

\begin{tabular}{llll}
\hline Periodo & Pequeño & Mediano & Grande \\
\hline Antes de & $\$$ & $\$$ & $\$$ \\
suspensión & $2.810,00$ & $3.880,00$ & $4.225,00$ \\
Durante & $\$-$ & $\$-$ & $\$-$ \\
suspensión & 380,00 & 803,00 & 150,00 \\
Después de & $\$$ & $\$$ & $\$$ \\
suspensión & $1.683,00$ & $1.970,00$ & $2.590,00$ \\
\hline
\end{tabular}

Fuente: elaboración propia (2021)

De la totalidad de usuarios que asisten a los gimnasios, los casos de sospechas de contagio por covid-19, alcanza un mínimo de porcentaje, apenas el $4 \%$, que corresponden a usuarios que, presentaron algún síntoma o se les detectó temperatura elevada mediante el uso de los protocolos de bioseguridad y que fueron reportados. 


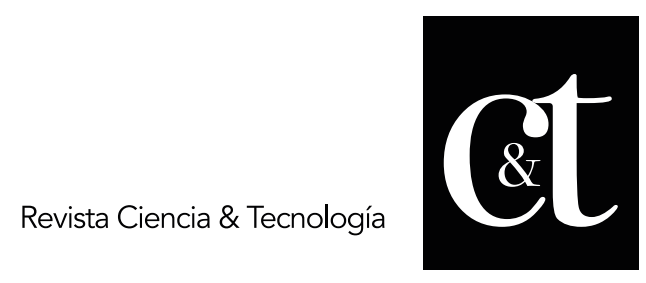

No. 32, 31 de octubre de 2021

ISSN impreso: 1390 - 6321

ISSN online: 2661 - 6734

Mientras que los casos confirmados de contagio, tan sólo alcanza el 1,34\% lo que significa que no todos los casos de sospechas, correspondieron a casos confirmados de contagio, incluso se infiere que los casos confirmados, el contagio no fue dentro de las instalaciones de los gimnasios, sino en reuniones sociales al exterior.

Tabla 2: Contagios por Covid 19

\begin{tabular}{lrrr}
\hline & $\begin{array}{r}\text { Pequeñ } \\
\text { o }\end{array}$ & $\begin{array}{r}\text { Median } \\
\text { o }\end{array}$ & $\begin{array}{r}\text { Grand } \\
\text { e }\end{array}$ \\
\hline $\begin{array}{l}\text { Número de usuarios } \\
\text { sospechas Covid }\end{array}$ & $0,84 \%$ & $4,07 \%$ & $4,84 \%$ \\
$\begin{array}{l}\text { Número de usuarios con } \\
\text { Covid }\end{array}$ & $0,84 \%$ & $1,02 \%$ & $1,34 \%$ \\
\hline
\end{tabular}

Fuente: elaboración propia (2021)

Se evidencia un bajo cumplimiento de las estrategias de inbound marketing, puesto que del $100 \%$ del total de cumplimiento, representados por 63 ítems, los gimnasios de pequeña capacidad alcanzaron el 52,4\%, los de mediana capacidad, obtuvieron un $50,0 \% ; y$, los gimnasios de gran capacidad alcanzaron el $42,5 \%$.

Entre las dimensiones que representan un mayor cumplimiento por parte de los gimnasios, se encuentra la consideración de objetivos en la creación de contenido, cuyo cumplimiento alcanzó el $83,33 \%$; por lo que se puede inferir que los contenidos publicados por los gimnasios, propician una imagen de autoridad en su nicho de mercado; además, los contenidos buscan que los clientes se identifiquen con la marca y su fidelización, así como diferenciarse de sus competidores; por otro lado, los gimnasios buscan contactar a clientes, ganar nuevos seguidores y generar confianza con la marca. Otra de las dimensiones que alcanzó mucho cumplimiento en la estrategia de inbound marketing por parte de los gimnasios, es la de los requisitos considerados en la creación de contenido de calidad, con un porcentaje de $75,69 \%$. 


\begin{tabular}{|c|c|c|c|c|c|c|c|}
\hline \multirow[b]{2}{*}{ No. } & \multirow[b]{2}{*}{ Dimensión } & \multirow[b]{2}{*}{ Ítems } & \multirow[b]{2}{*}{ Peso } & \multirow{2}{*}{$\begin{array}{l}\% \\
\text { Ponderado }\end{array}$} & \multicolumn{3}{|c|}{$\%$ Alcanzado } \\
\hline & & & & & Pequeño & Mediano & Grande \\
\hline 1 & $\begin{array}{l}\text { Canales utilizados para hacer presencia } \\
\text { en internet }\end{array}$ & 8 & 32 & $12,70 \%$ & $4,80 \%$ & $5,20 \%$ & $4,80 \%$ \\
\hline 2 & $\begin{array}{l}\text { Tipos de contenidos utilizados para } \\
\text { generar tráfico }\end{array}$ & 12 & 48 & $19,05 \%$ & $8,70 \%$ & $11,50 \%$ & $6,30 \%$ \\
\hline 3 & $\begin{array}{l}\text { Objetivos que considera en la creación } \\
\text { del contenido }\end{array}$ & 7 & 28 & $11,11 \%$ & $8,70 \%$ & $9,50 \%$ & $9,50 \%$ \\
\hline 4 & $\begin{array}{l}\text { Requisitos considerados en la creación de } \\
\text { contenido de calidad }\end{array}$ & 12 & 48 & $19,05 \%$ & $15,90 \%$ & $14,70 \%$ & $12,70 \%$ \\
\hline 5 & $\begin{array}{l}\text { Factores que generan autenticidad a la } \\
\text { marca }\end{array}$ & 11 & 44 & $17,46 \%$ & $2,40 \%$ & $1,60 \%$ & $1,20 \%$ \\
\hline 6 & $\begin{array}{l}\text { Con el fin de recabar datos de tus } \\
\text { posibles clientes, ¿qué herramientas } \\
\text { utilizas? }\end{array}$ & 4 & 16 & $6,35 \%$ & $2,40 \%$ & $1,60 \%$ & $1,20 \%$ \\
\hline 7 & $\begin{array}{l}\text { Segmentación en la promoción del } \\
\text { contenido }\end{array}$ & 6 & 24 & $9,52 \%$ & $6,00 \%$ & $3,50 \%$ & $4,40 \%$ \\
\hline 8 & Marketing y Embudo de Ventas & 3 & 12 & $4,76 \%$ & $3,50 \%$ & $2,40 \%$ & $2,40 \%$ \\
\hline & Total & 63 & 252 & $100,00 \%$ & $52,40 \%$ & $50,00 \%$ & $42,50 \%$ \\
\hline
\end{tabular}

Fuente: elaboración propia (2021)

Por otro lado, existen dimensiones con muy baja calificación, una de éstas son los factores que generan autenticidad a la marca, alcanzando apenas el 9,85\%, observando los resultados, no se evidencia ninguna actividad donde participen influencers en la creación, colaboración o distribución de contenidos; poco o nada se usan formularios o encuestas personalizadas para obtener información, ni se suele usar personal brand para atraer usuarios, con poca frecuencia, los contenidos son enriquecidos sobre una base científica, ni ofrecen valor a los usuarios, con frecuencia regular se crean historias en vivo donde se permite la participación de los usuarios - storydoing; asimismo, no todos los contenidos digitales son originales, y con poca frecuencia evidencian información clara y real de lo prometido por la marca; se nota un poca más de actividad en contar historias reales - Storytelling; y se trata de usar una línea gráfica, con logos, hashtag, slogan a nivel interno y externo. La mayor frecuencia se nota en considerar buenas prácticas de recolección de los datos proporcionados por los usuarios. 


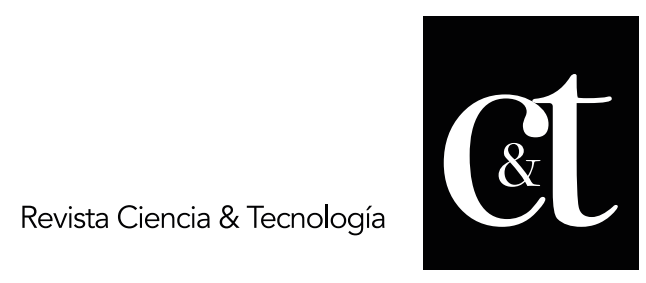

No. 32, 31 de octubre de 2021

ISSN impreso: 1390 - 6321

ISSN online: 2661 - 6734

Una dimensión de gran importancia a la hora de convertir los potenciales clientes, es la que se refiere a las herramientas utilizadas con el fin de recabar datos de estos clientes potenciales, esta dimensión apenas alcanzó el 27,08\%. Entre las actividades en redes sociales, los gimnasios no publicaron formularios de suscripción a través de prueba gratuita o demostración de un servicio; aunque con frecuencia si publican formularios de registro mediante link y formularios de suscripción en contenido digital para redes sociales.

Una de las dimensiones de estudio de gran relevancia, es el uso de canales para hacer presencia en internet, que, de acuerdo a los resultados obtenidos, los gimnasios no suben contenidos a YouTube por cuanto no registran cuentas creadas en esta plataforma, al igual que en Twitter ni en página web o blog alguno; una actividad poco frecuente, es la que realizan en Google Plus. Entre otros canales utilizados, se encuentran Facebook, Instagram y WhatsApp. En la dimensión de estudio, que se refiere a los tipos de contenidos utilizados para generar tráfico en internet, se pudo observar que esta dimensión alcanzó un $46,53 \%$, donde no se evidencia contenido tales como artículos, noticias o contenidos de terceros, muy poco contenido sobre memes o audios; en mayor proporción se nota contenidos como guías o tutoriales, y con mayor frecuencia, contenidos en vivo, infografías, posts; y, casi siempre suben videos, historias y fotografías.

\section{Cálculo ROI}

En este apartado, se presentan los resultados del cálculo del retorno sobre la inversión - ROI de las campañas de inbound marketing gestionadas por los gimnasios, para ello se sigue el siguiente proceso: se calcula el Valor de la Relación del Cliente - CLV, considerando una retención por 6 meses, mediante la siguiente fórmula:

$C L V=P V M \times T R$

Donde:

CLV $=\quad$ Valor de la Relación del Cliente

$\mathrm{PVM}=$ Promedio del Valor de Membresía

$\mathrm{TR}=$ Tiempo de retención de clientes, definido para este ejercicio en 6 meses. Una vez calculado el CLV, se calcula el Costo de Adquisición de Clientes - CAC, mediante la siguiente fórmula:

$\mathrm{CAC}=\mathrm{PTMO} / \mathrm{ANPC}$

Donde:

CAC $=$ Costo de Adquisición de Clientes

PTMO = Presupuesto total de Marketing Online

ANPC $=$ Aceptable Número positivo de Clientes de ROI.

Para calcular el ROI, fue utilizada la siguiente fórmula:

$\mathrm{ROI}=((\mathrm{CLV} \times \mathrm{ANPC})-\mathrm{PTMO}) / \mathrm{PTMO}$ 


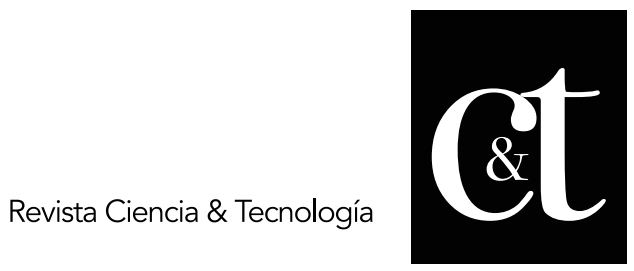

No. 32, 31 de octubre de 2021

ISSN impreso: 1390 - 6321

ISSN online: 2661 - 6734

Tabla 4: Cálculo del ROI

\begin{tabular}{lrrr}
\hline \multicolumn{1}{c}{ Cálculo ROI } & \multicolumn{2}{c}{ Tipo de Gimnasio } \\
& Pequeño & Mediano & \multicolumn{1}{c}{ Grande } \\
\hline Promedio Valor de Membresía & $\$ 20,00$ & $\$ 30,00$ & $\$ 40,00$ \\
Presupuesto total de Marketing Online & $\$ 55,00$ & $\$ 390,00$ & $\$ 588,00$ \\
Valor de la Relación del Cliente CLV $(6$ & $\$ 120,00$ & $\$ 180,00$ & $\$ 240,00$ \\
Meses) & & & \\
$\begin{array}{l}\text { Aceptable Número positivo de Clientes } \\
\text { de ROI }\end{array}$ & 22 & 73 & 99 \\
Costo de Adquisición de Clientes CAC & $\$ 2,50$ & $\$ 5,34$ & $\$ 5,94$ \\
\hline ROI & $\$ 47,00$ & $\$ 32,69$ & $\$ \mathbf{3 9 , 4 1}$ \\
\hline
\end{tabular}

Fuente: elaboración propia (2021)

El retorno sobre la inversión por la gestión de estrategias de inbound marketing, sigue siendo muy rentable en los gimnasios, aún en los gimnasios de pequeña capacidad y con una inversión inferior en relación a los de mayor capacidad, el ROI es superior, por lo que se puede inferir que se están desarrollando actividades en redes sociales muy efectivas, que permite que las conversiones se realicen con mayor facilidad. En los casos de los gimnasios de mayor capacidad, el retorno sobre la inversión puede alcanzar casi los $\$ 40,00$, un valor muy rentable para estos negocios, aunque se debe considerar que en el ROI no se considera los costos generados por el servicio brindado, puesto que el cálculo se lo realiza en función del presupuesto destinado para las campañas de marketing digital.

\section{Conclusiones}

Las estrategias de inbound marketing aportan significativamente en la reactivación económica de los gimnasios de la ciudad de Babahoyo, luego de haber sido gravemente afectados por casusa de la pandemia por covid-19, el proceso de reactivación ha sido progresivo y aunque hasta febrero de 2021 existen limitaciones dispuestas por los organismos de control, en el ejercicio del cálculo del Retorno sobre la Inversión de la estrategia de inbound marketing, se evidenció una muy buena rentabilidad, incluso en los gimnasios de pequeña capacidad se observó una mayor rentabilidad con respecto a los gimnasios más grandes.

Las campañas efectuadas, se enfocan en devolver la confianza a los clientes, para que puedan desarrollar sus entrenamientos en instalaciones seguras que cumplen con los protocolos de bioseguridad y los requerimientos de los organismos de control.

Las actividades enfocadas al trabajo en redes sociales, que agrupan las actividades más efectivas, una es la consideración de objetivos en la creación de contenidos, cuyo cumplimiento alcanzó el $83,33 \%$; por lo que se concluye que los contenidos publicados por los gimnasios, propician una imagen de autoridad en su nicho de mercado, además, los contenidos buscan que los clientes se identifiquen con la marca y su fidelización, así como diferenciarse de sus competidores, por otro lado, los gimnasios buscan contactar a clientes, ganar nuevos seguidores y generar confianza con la marca. 


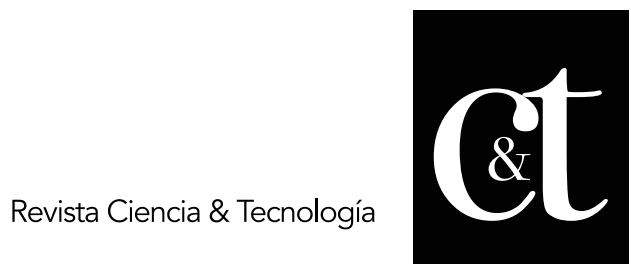

No. 32, 31 de octubre de 2021

ISSN impreso: 1390 - 6321

ISSN online: 2661 - 6734

Otra de las sub-dimensiones que alcanzó mucho cumplimiento en la estrategia de inbound marketing por parte de los gimnasios, es la de los requisitos considerados en la creación de contenido de calidad, con un porcentaje de $75,69 \%$, por lo que se concluye que los gimnasios crean contenidos que son pensados para una audiencia específica, con un objetivo concreto, útil y valioso para quien lo recibe, que tiene credibilidad y es natural, que merece ser compartido, logrando cautivar y entretener al receptor, con contenidos bien redactados y profesionalmente presentados, de relevancia respecto a los objetivos planteados, abordando temas en profundidad, demostrando conocimiento del emisor, evitando en lo posible el lenguaje promocional o corporativo, que soluciona problemas y responde cuestiones concretas.

\section{Referencias bibliográficas}

Alguacil, M., Crespo Hervás, J., \& Pérez Campos, C. (2020). Análisis sociodemográfico de la percepción de marca en un servicio deportivo público: del target al buyer persona. RETOS. Nuevas Tendencias En Educación Física, Deporte y Recreación, 2041(37), 139-146.

Banco Central del Ecuador. (2020). Producto interno bruto por industria. Quito. Recuperado de https://contenido.bce.fin.ec/documentos/PublicacionesNotas/Catalogo/IEMens $\mathrm{ual} / \mathrm{m} 2020 / \mathrm{IEM}-432$-e.xlsx

Chase, H. (2020). Customer Acquisition Cost: The One Metric That Can Determine Your Company's Fate. Retrieved January 10, 2021, from https://neilpatel.com/blog/customer-acquisition-cost/

Cintell. (2019). The Right Customer at the Right time. Retrieved from https://www.cintell.net/the-right-customer-at-the-right-time/

Hernández, R., Fernández, C. y Baptista, P. (2014). Metodología de la investigación. Metodología de la investigación (Sexta Edic). McGraw-Hill

Hubspot. (2020). What Is the Buyer's Journey? Retrieved September 28, 2020, from https://blog.hubspot.com/sales/what-is-the-buyers-journey

INEC. (2012). Hábitos de lectura en Ecuador. Retrieved from http://www.inec.gob.ec/documentos_varios/presentacion_habitos.pdf

Instituto Nacional de Estadística y Censos. (2015). Encuesta Calidad De Vida 2015. Compendio de Resultados de La Encuesta de Condiciones de Vida ECV 2014 (Noviembre 2013 - Octubre 2014), 1(1), 197. Retrieved from https://www.ecuadorencifras.gob.ec/documentos/webinec/ECV/ECV_2015/documentos/ECV COMPENDIO LIBRO.pdf

Lindblom, M., \& Andreasson, A. (2019). Bachelor Thesis Bachelor's Programme in International Marketing, 180 credits Inbound marketing from a B2B-perspective Independent Project in Business. Halmstad University.

Loredana, P.-B. (2016). Inbound Marketing - the most important digital marketing strategy. Boletín de La Universidad Transilvania de Brasov. Serie V: Ciencias Económicas, 9(2), 61-68. Retrieved from https://www.semanticscholar.org/paper/Inbound-Marketing-the-mostimportant-digitalLoredana/486b56f34dc6629d7ed74f054b857b56f74a52d6?p2df

Nuviala Nuviala, R., Teva Villen, M. R., Pérez Ordás, R., Grao Cruces, A., Tamayo Fajardo, J. A., \& Nuviala Nuviala, A. (2014). Segmentación de usuarios de servicios deportivos. RETOS. Nuevas Tendencias En Educación Física, Deporte y Recreación, (25), 90-94. 


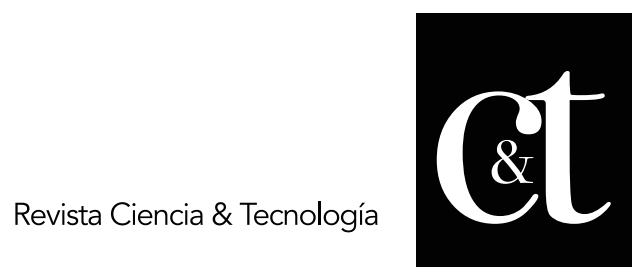

No. 32, 31 de octubre de 2021

ISSN impreso: 1390 - 6321

ISSN online: 2661 - 6734

OMS. (2017). 10 datos sobre la actividad física. Retrieved from https://www.who.int/features/factfiles/physical_activity/es/

Raatikainen, L. (2018). Measuring Inbound Marketing. Haaga-Helia. University of Applied Sciences.

Repoviené, R. (2017). Role of content marketing in a value creation for customer context: theoretical analysis. International Journal on Global Business Management and Research. Tamilnadu, India: Rajalakshmi Educational Trust, 2017, Vol. 6, Iss. 2.

Teba Garrido, C. (2016). Diferencias entre Inbound Marketing y Outbound Marketing. Retrieved August 6, 2020, from https://comunidad.iebschool.com/elinboundestademoda/2016/11/25/diferenci as-inbound-marketing-outbound-marketing/

Thi Thuy, N. B. (2020). Inbound Marketing Plan for Small \& Medium-Sized Businesses. Metropolia University of Applied Sciences. 